\title{
Wie Politik im Spitalalltag ankommt
}

\author{
Im September fanden zwei Veranstaltungen* statt, die sich mit den Auswirkungen \\ der neuen Spitalfinanzierung auf die Qualität der Gesundheitsversorgung beschäftig- \\ ten. Beim Besuch der beiden Tagungen wurde deutlich, dass der «politische Wille» \\ selten eins zu eins in der Praxis der Versorgungsstrukturen ankommt.
}

Anna Sax

\section{Die Auswirkungen der politischen Rahmenbedingungen auf die Planungs- und Strategieentscheide} der Leistungserbringer wurden sichtbar gemacht.

*- SGfM Tagung «Qualitätsmedizin Schweiz Teil I: SwissDRG, ANQ, Qualitätslabel, Versorgungsforschung Welchen Beitrag leisten die einzelnen Akteure?», 14.9.2012 in Bern

PGMM Kongress «Spital der Zukunft: Strategie - Prozesse Infrastruktur», 26.9.2012 in Winterthur
Wenn man Nationalrätin Ruth Humbel glauben darf, dann hat sich der Gesetzgeber Ende 2007, als im Parlament der Entscheid für die neue Spitalfinanzierung fiel, etwas anderes vorgestellt als das, was die Akteure heute daraus machen. In ihrem einleitenden Referat an der Tagung der Schweizerischen Gesellschaft für Medizincontrolling SGfM fasste sie das Befremden in

Worte, das sich unter Gesundheitspolitikern breitmacht, die sich von der leistungsorientierten DRGFinanzierung grosse Verbesserungen erhofft hatten und nun feststellen müssen, dass sich in den Köpfen der Gesundheits- und Spitaldirektorinnen nicht viel verändert hat. Das Bundesparlament wollte mehr Transparenz, mehr Wettbewerb und mehr Qualität. Doch die Kantone untergraben die Absichten der Bundespolitik, indem sie spitalindividuelle Baserates bewilligen, Mengen begrenzen und Spitallisten weiterhin nach politischen Kriterien erlassen. Die Spitäler sind nicht willens oder nicht in der Lage, die notwendigen Daten für aussagekräftige Betriebsvergleiche über die medizinische Qualität zu liefern. «Die Kantone kontrollieren Restaurantküchen schärfer als Spitäler», monierte Comparis-Sprecher Felix Schneuwly in seinem darauffolgenden Referat. Mangels Transparenz würde die - ebenfalls bundespolitisch gewollte - freie Spitalwahl für die Patientinnen und Patienten zur Farce.

Die enttäuschten Bundespolitikerinnen und -politiker hätten vielleicht gut daran getan, die Veranstaltung zu besuchen, die Ende September im Kantonsspital Winterthur stattfand. Hier wurde deutlich, wie die Spitalverantwortlichen ticken, beziehungsweise, wie sie versuchen, aus den politischen Vorgaben das Optimum für ihre Betriebe herauszuholen. Und es zeigte sich, wie schwierig es ist, Politik und wirtschaftliche Anreize in Einklang zu bringen.
Beim PGMM-Kongress ging es vordergründig um die Frage, wie sich die neue Spitalfinanzierung auf die Entwicklung der Spitalinfrastrukturen auswirkt. Die auf Medizinalplanung, Gebäudetechnik und Immobilienmanagement spezialisierte Firma PGMM lud dazu Spitalmanager, Immobilienfachleute und weitere Spitalexpertinnen ein. Hinter der Veranstaltung stand ein Konzept, das über finanz- und logistikorientierte Sichtweisen hinaus das gesundheitspolitische Umfeld miteinbeziehen wollte. In den Referaten wurde der Bogen gespannt von der Finanzierung der Gesundheitsversorgung bis hin zum Spitalalltag. Betrachtungen über Spardruck und Nutzenorientierung aus volkswirtschaftlicher Sicht machten den Anfang. Es folgten Empfehlungen darüber, welche Strategien die Spitäler in der Positionierung der Leistungsangebote unter DRG verfolgen können. Weiter ging es mit Reflexionen über Prozesse und Infrastrukturen bis hin zur praktischen Bedeutung der DRGs im Spitalalltag.

Schon fast als Anleitung zum «Rosinenpicken» könnten die Ausführungen des Siemens-Gesundheitsexperten Christoph Napierala aufgefasst werden.

\section{Die Autorin als Referentin}

$B K$ - Anna Sax, Gesundheitsökonomin und Autorin dieses Beitrags, hielt am PGMM-Kongress das Eröffnungsreferat. Nachfolgend eine kurze Zusammenfassung:

Sparen als politische Strategie sei keine gute Wahl, erklärte Anna Sax: «Wer nur ans Sparen denkt, denkt eindimensional und sieht meist nicht über den eigenen Tellerrand hinaus.» Statt am Sparen sollten wir uns laut Sax stärker an der Wirtschaftlichkeit orientieren, denn wer wirtschaftlich denke, komme nicht darum herum, auch den Patientennutzen in seine Überlegungen miteinzubeziehen. Die Patientenorientierung messe sich selten an teuren Therapien, jedoch immer an Zuwendung, korrekter Information und Kooperation. Mit einer konsequenten Anwendung von HTA-Verfahren für teure medizinische Leistungen und integrierten Versorgungsmodellen für chronisch Kranke seien bessere Qualität und wirtschaftliche Vorteile unter einen Hut zu bringen. 


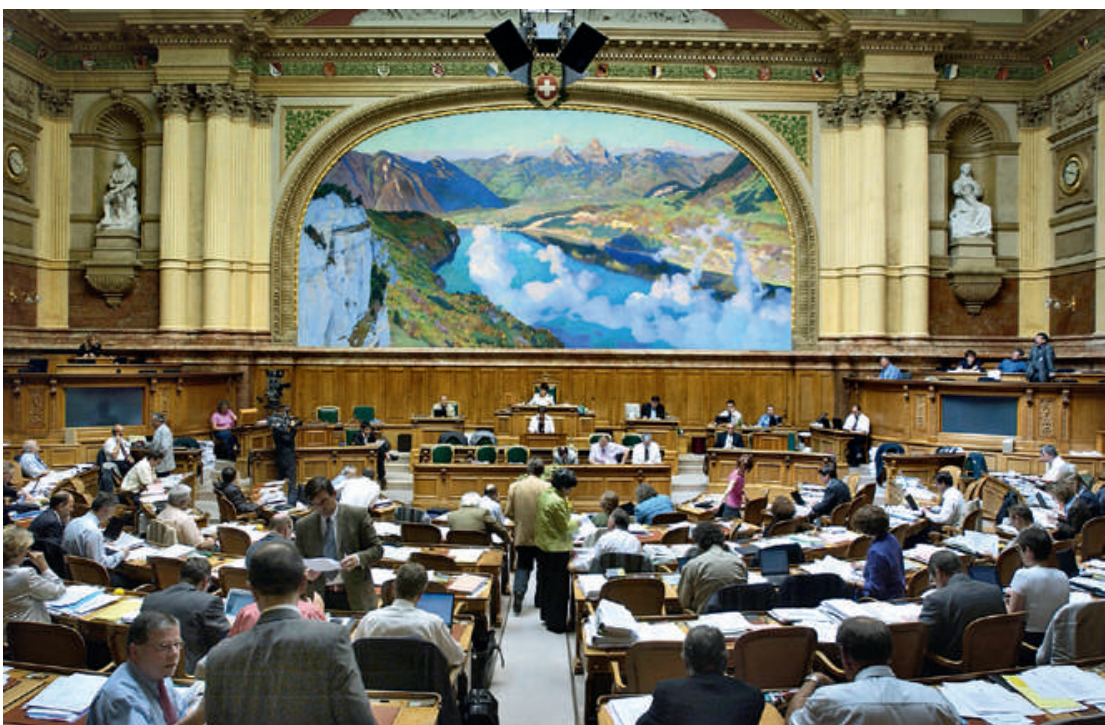

Was sich die Politik vorgestellt hat, kommt nicht immer eins zu eins im Spital an.

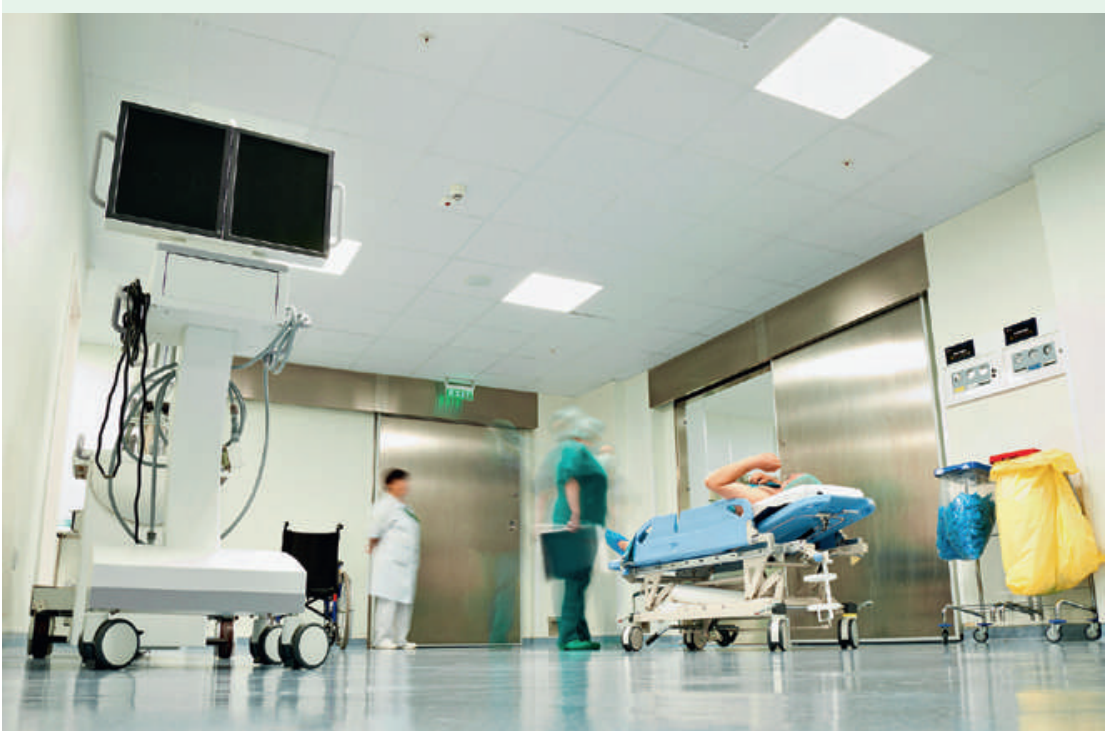

Wer im DRG-Zeitalter Wettbewerbsvorteile erzielen wolle, müsse die Fallzusammensetzung optimieren und Leistungsaufträge, die nicht kostendeckend $\mathrm{zu}$ erfüllen seien, an den Kanton zurückgeben, so Napierala. Der Fehler liege beim System, nicht bei den Spitälern, wenn dadurch gewisse Leistungen nicht mehr genügend abgedeckt würden. Es sei Aufgabe der Politik, auf drohende Versorgungslücken zu reagieren - nicht mit höheren Baserates, aber mit Sonderentgelten für besonders komplexe Fälle.

Unter dem Titel «Prozessgerechte Infrastruktur infrastrukturgerechte Prozesse» versuchte Valentin Simonett, Bereichsleiter Medizinalplanung bei PGMM
Schweiz AG, Wirtschaftstheorie und Spitalpraxis miteinander in Einklang zu bringen. «Je einfacher der Prozess, desto klarer die dazugehörige Infrastruktur», fasste Simonett die unterschiedlichen Anforderungen an eine prozessgerechte Infrastruktur zusammen, und illustrierte das Gesagte mit dem Bild einer Sprungschanze. Der zu dieser Infrastruktur gehörige Prozess geht so: Man stellt sich oben hin und fährt hinunter. Entsprechend einfach ist die Infrastrukturplanung in diesem Fall. Im Spital dagegen ist die Infrastrukturplanung eine sehr viel komplexere Angelegenheit, allein schon wegen der unterschiedlichen Lebenszyklen von Bauten, Einrichtungen und Technik. Auf der anderen Seite sind infrastrukturgerechte Prozesse essentiell - dafür braucht es Freiräume und Reserven, die Anpassungen möglich machen.

Mit Christoph Scheu meldete sich zum Abschluss ein deutscher Arzt zu Wort, der geradezu euphorisch die neuen Möglichkeiten pries, die sich dank der DRG-Finanzierung für die Strategie eines Spitals eröffneten. «DRG haben nirgends auf der Welt etwas eingespart. Aber wir wissen endlich, was läuft!», begeisterte sich der Geschäftsführer eines grösseren katholischen Krankenhauses im bayerischen Straubing, und: «DRG schaffen eine brutale Transparenz.» Davor hätten die Ärzte noch zu viel Angst. Er wolle die Ärztinnen und Ärzte in seinem Krankenhaus dazu ermuntern, die DRG als «eine phantastische Möglichkeit zur Analyse und Steuerung des medizinischen Geschehens» zu sehen. Dafür sei es aber entscheidend, dass sich die Ärzte mitverantwortlich fühlten für die Codierung und für die Umsetzung der neu gewonnenen Erkenntnisse. Die Arbeit mit Qualitätsindikatoren aus DRG-Routinedaten könne nicht früh genug aufgenommen werden, schloss Scheu seine Ausführungen, damit Spital und Patienten als Gewinner herauskommen.

Der erste PGMM-Kongress dauerte nur einen halben Tag, und doch gelang es den Organisatoren, die Auswirkungen der politischen Rahmenbedingungen auf die Planungs- und Strategieentscheide der Leistungserbringer - und damit letztlich auf die Ärztinnen, Pflegenden und Patienten - sichtbar zu machen. Zudem ermöglichte die Veranstaltung einen guten Einblick in das Denken der Spitalmanager, Spitalbauer, Zulieferer und Berater. Und mit den bitteren Worten von Nationalrätin Ruth Humbel in den Ohren wird deutlich, mit wie vielen Unwägbarkeiten der Weg vom «politischen Willen» zum konkreten Spitalalltag gespickt ist. 